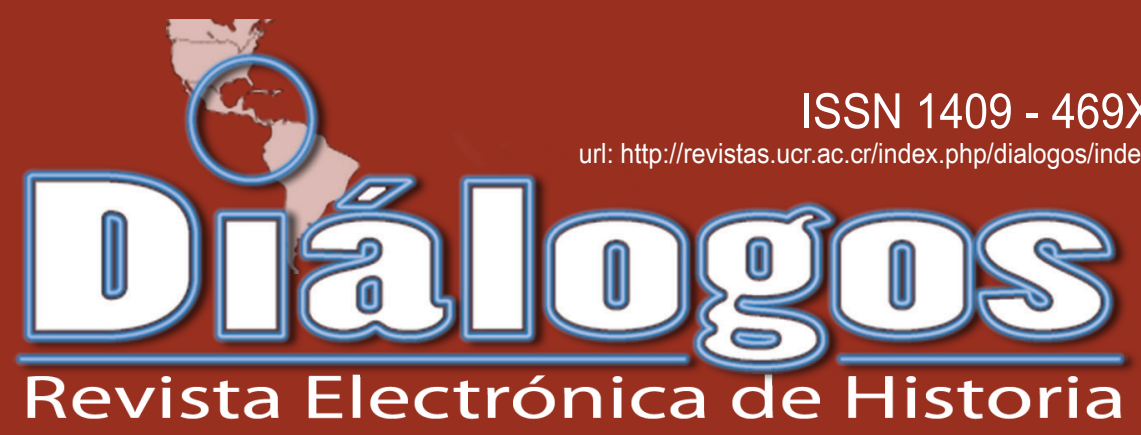

Escuela de Historia. Universidad de Costa Rica Volumen 14 especial en homenaje a Victor Hugo Acuña. Octubre 2013



Director de la Revista: Dr. Juan José Marín Hernández juan.marinhernandez@ucr.ac.cr

Editor académico: Dr. Ronny Viales Hurtado - ronny.viales@ucr.ac.cr Editor técnico: M.Sc. Marcela Quirós G. - marcela.quiros@ucr.ac.cr 



\section{Miembros del Consejo Editorial:}

Dr. Juan José Marín Hernández, Catedrático. Director del Centro de Investigaciones Históricas de América Central. Universidad de Costa Rica. Costa Rica. juan. marin@ucr.ac.cr

Dr. Ronny Viales Hurtado. Catedrático. Historia Económica y Social. Universidad de Costa Rica. Director de la Escuela de Historia. Costa Rica. ronny. viales@ucr.ac.cr

Dr. David Díaz Arias: Catedrático. Historia Política, Director del posgrado de Historia y Docente de la Escuela de Historia, Universidad de Costa Rica, Costa Rica.david.diaz@ucr.ac.cr

MSc. Francisco Enríquez. Historia Social. Universidad de Costa Rica. Costa Rica. francisco.enriquez@ucr. ac.cr

Dra. Ana María Botey. Historia de los movimientos sociales. Universidad de Costa Rica. Costa Rica. abotey@gmail.com

\section{Miembros del Consejo Asesor Internacional:}

Dr. José Cal Montoya. Universidad de San Carlos de Guatemala.Guatemala. jecalm@correo.url.edu.gt

Dr. Juan Manuel Palacio. Universidad Nacional de San Martín. Argentina.jpalacio@unsam.edu.ar

Dr. Eduardo Rey. Universidad de Santiago de Compostela. España. ereyt@usc.es

Dr. Heriberto Cairo Carou. Departamento de Ciencia Política y de la Administración III Universidad Complutense de Madrid. España. hcairoca@cps.ucm.es

Dra. Rosa de la Fuente. Departamento de Ciencia Política y de la Administración III Universidad Complutense de Madrid. España. rdelafuente@cps. ucm.es

Dr. Javier Franzé. Departamento de Ciencia Política y de la Administración III Universidad Complutense de Madrid. España. javier.franze@cps.ucm.es

Dr. Jaime Preciado Coronado Departamento de Estudios Ibéricos y Latinoamericanos. Universidad de Guadalajara. México. japreco@hotmail.com
Dr. Gerónimo de Sierra. Vicerrector de la Universidade Federal da Integração Latino-Americana (UNILA) y Departamento de Sociología, Facultad de Ciencias Sociales de la Universidad de la República. Uruguay. geronimo@fcs.edu.uy

Dr. Antonio Palazuelos. Departamento de Ciencia Política y de la Administración III - Universidad Complutense de Madrid. España. palazuelosa@cps. ucm.es

Dr. Werner Mackenbach. Universidad Potsdam. Alemania. werner.mackenbach@uni-potsdam.de

Dr. Guillermo Castro. Ciudad del Saber Panamá. Panamá.gcastro@cdspanama.org

Dra. Natalia Milanesio. University of Houston. Estados Unidos.nmilane2@Central.UH.EDU

Dr. Ricardo González Leandri. Consejo Superior de Investigaciones Científicas - España. España. rgleandri@gmail.com

Dra. Mayra Espina. Centro de Estudios Psicológicos y Sociológicos, La Habana. Cuba.mjdcips@ceniai.inf.cu

Dra. Montserrat Llonch. Departamento de Economía e Historia Económica Universidad Autónoma de Barcelona. España.montserrat.llonch@uab.es

Dra. Estela Grassi. Universidad de Buenos Aires. Argentina. estelagrassi@gmail.com

Dra. Yolanda Blasco. Universidad de Barcelona. España. yolandablasco@ub.edu

Dr. Alfredo Falero. Departamento de Sociología. Universidad de la República. Uruguay. alfredof@adinet. com.uy

\section{Portada:}

Fotografía de Anel Kenjekeeva, Oficina de Divulgación Universidad de Costa Rica, publicada en http://www. ucr.ac.cr/noticias/2012/09/13/escuela-de-historia-rindehomenaje-a-victor-hugo-acuna.html

\section{Equipo Técnico Editorial:}

$\begin{array}{ll}\text { Editora Técnica: } & \text { M.Sc. Marcela Quirós Garita. } \\ & \text { marcela.quiros@ucr.ac.cr } \\ \text { Diagramación: } & \text { Cindy Chaves Uribe } \\ \text { Asistentes: } & \begin{array}{l}\text { Pablo Hurtado Granados } \\ \text { Maureen Méndez Montero }\end{array}\end{array}$


"Diálogos Revista Electrónica de Historia" se publica desde octubre de 1999.

\section{Diálogos está en los siguientes repositorios:}

Dialnet

http://dialnet.unirioja.es/servlet/

revista?tipo_busqueda=CODIGO\&clave_revista $=3325$

Latindex

http://www.latindex.unam.mx/larga.php?opcion=1\&folio=12995;

\section{UCRindex}

http://www.revistas.ucr.ac.cr/

\section{Scielo}

http://www.scielo.cl/

\section{eRevistas}

http://www.erevistas.csic.es/

\section{REDALYC}

http://redalyc.uaemex.mx/src/inicio/FrmBusRevs2.jsp?iEdoRev=2\&cvepai=11;

\section{LANIC}

http://lanic.utexas.edu/la/ca/cr/indexesp.html

Repositorio de Revistas Universidad de Costa Rica http://www.latindex.ucr.ac.cr/

Directorio y recolector de recursos digitales del

Ministerio de Cultura de España

http://roai.mcu.es/es/inicio/inicio.cmd

DOAJ Directory of open access \& Hybrid journals

http://www.doaj.org/doaj?func=byTitle\&hybrid=1\&query=D

Biblioteca de Georgetown

http://library.georgetown.edu/newjour/d/msg02735.html

Asociación para el Fomento de los Estudios Históricos

en Centroamérica

http://afehc. apinc.org/index.php?action=fi_aff\&id=1774

Universidad de Saskatchewan, Canadá

https://library.usask.ca/ejournals/view/1000000000397982

\section{Monografias}

http://www.monografias.com/Links/Historia/more12.shtml

\section{Hispanianova}

http://hispanianova.rediris.es/general/enlaces/hn0708.htm

Universidad del Norte, Colombia

http://www.uninorte.edu.co/publicaciones/memorias/enlaces.htm

Universidad Autónoma de Barcelona

http://seneca.uab.es/historia/hn0708.htm

Repositorio Invenia - Gestión del Conocimiento

http://www.invenia.es/oai:dialnet.unirioja.es:ART0000086144

\section{Enlace Académico}

http://www.enlaceacademico.org/biblioteca/

revistas-en-formato-digital-centroamerica/

\section{Electronic Resources}

http://sunzi1.lib.hku.hk/ER/detail/hkul/3987318

Revistas académicas en texto completo

http://web.prw.net/ vtorres/
Diálogos se anuncia en las siguientes

instituciones y sitios académicos:

Maestroteca

http://www.maestroteca.com/detail/553/dialogos-revista-electronica-de-historia.html

Biblioteca de Georgetown

http://library.georgetown.edu/newjour/d/msg02735.html

Asociación para el Fomento de los Estudios Históricos en Centroamérica

http://afehc. apinc.org/index.php?action=fi_aff\&id=1774

Universidad de Saskatchewan, Canadá

https://library.usask.ca/ejournals/view/1000000000397982

\section{Monografias}

http://www.monografias.com/Links/Historia/more12.shtml

\section{Hispanianova}

http://hispanianova.rediris.es/general/enlaces/hn0708.htm

Universidad del Norte, Colombia

http://www.uninorte.edu.co/publicaciones/memorias/enlaces.htm |

Universidad Autónoma de Barcelona

http://seneca.uab.es/historia/hn0708.htm

Repositorio Invenia - Gestión del Conocimiento

http://www.invenia.es/oai:dialnet.unirioja.es:ART0000086144

\section{Enlace Académico}

http://www.enlaceacademico.org/biblioteca

revistas-en-formato-digital-centroamerica/

\section{Electronic Resources}

http://sunzi1.lib.hku.hk/ER/detail/hkul/3987318

Revistas académicas en texto completo

http://web.prw.net/ vtorres/

La revista electrónica Diálogos es financiada por

Vicerrectoría de Investigación de la Universidad de Costa Rica
Dialnet - eRevistas

- UCRindex - Latindex -

REDALYC - DOJAC - Directorio

y recolector de recursos

digitales del Ministerio de

Cultura de España

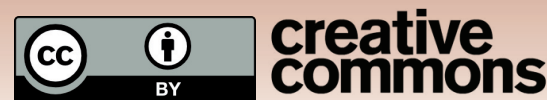




\title{
EL IMPACTO SOCIAL Y ECONÓMICO GENERADO POR EL TURISMO DE LOS CRUCEROS QUE VISITAN LA CIUDAD DE PUNTARENAS
}

\author{
THE SOCIAL AND ECONOMIC IMPACT OF CRUISE TOURISM IN \\ PUNTARENAS (COSTA RICA)
}

María Lisbeth Mora Elizondo

\begin{abstract}
Palabras claves
Impacto social, impacto económico, turismo de cruceros, gobierno local, gestión turística, Puntarenas.
\end{abstract}

\section{Keywords}

Social impact, economic impact, Cruise Ship Tourism, Local government, tourism management, Puntarenas.

Fecha de recepción: 17 de mayo, 2013 - Fecha de aceptación: 8 de agosto 2013

\begin{abstract}
Resumen
Esta investigación analiza el impacto social y económico generado por la afluencia turística proveniente de los cruceros que llegan a la ciudad de Puntarenas, temporada en la que hay mucho movimiento en la zona e incluso se observa un ambiente mucho más pintoresco y atractivo. Además, se indaga sobre las actividades que se realizan durante la visita de los cruceros, la acción del gobierno local, y la existencia de dificultades o necesidades para el mejoramiento de la gestión turística de este sector y sus posibles soluciones, de acuerdo con el criterio de las personas involucradas en la atención de los turistas.
\end{abstract}

\begin{abstract}
This investigation took into account the Cruise Ship Tourism which arrives in Puntarenas City, season in which there is a lot of movement in the area and even it is shown more colorful and attractive. In this study, it will investigate about the social and economic impact generated precisely by the cruise ship tourism. Moreover, it was inquired into the activities which take place during the visit of the cruise ship, the action of the local government, as well as the presence of difficulties or needs to improve the tourism management in this sector and possible solutions according to the people involved.
\end{abstract}




\title{
EL IMPACTO SOCIAL Y ECONÓMICO GENERADO POR EL TURISMO DE LOS CRUCEROS QUE VISITAN LA CIUDAD DE PUNTARENAS
}

\author{
María Lisbeth Mora Elizondo
}

\section{HISTORIA DE PUNTARENAS}

Para referirse a la ciudad de Puntarenas es indispensable tomar en cuenta a sus primeros pobladores, los cuales vivieron en esta área antes y durante el período de la colonia; por ejemplo, de acuerdo con el Instituto Costarricense de Puertos del Pacífico (INCOP), los grupos indígenas que poblaron la zona fueron los Bruncas, Coctos y Buricas, que al igual que el resto del país sufrieron las consecuencias de la colonización en los años 1522. Cabe destacar que hubo otro grupo de indígenas que vivió en esta área, específicamente por Herradura, y eran conocidos como el grupo Huetar y posteriormente, como los Quepos. El nombre de Puntarenas se originó en la topografía del lugar, ya que los ríos, los vientos y la corriente del Rio Barranca fueron formando una lengüeta con lodo y arena, es así como en el año 1722 le llamaron Puerto de Arenas (Instituto Costarricense de Puertos del Pacífico, INCOP, 2010).

De acuerdo con la reseña histórica de la Municipalidad de Puntarenas, en el año 1847 Puntarenas tenía gran importancia en las exportaciones de Café a Inglaterra y Chile, lo cual fue impulsado por la construcción del ferrocarril al Pacífico en 1910. También, se incrementaron las actividades portuaria, ferrocarrilera y turística. Cabe destacar que la Carretera Interamericana se construyó en el año 1950 y posteriormente la Carretera Alberto Echandi a la ciudad (Municipalidad de Puntarenas, 2012).

Entre las principales actividades económicas destacan la pesca arte-

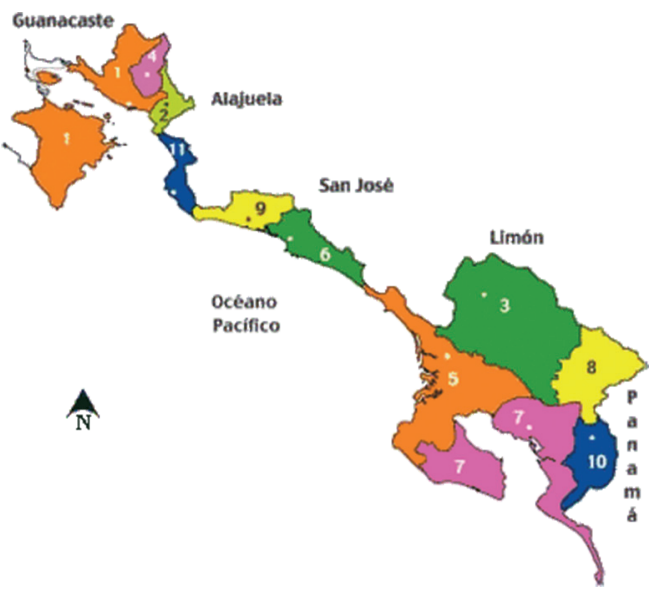

Figura 1. Mapa de la Provincia de Puntarenas Fuente: Página Web del Instituto Costarricense de Puertos del Pacífico (2010). sanal e industrial, la agricultura de los principales granos, frutas y verduras y la ganadería de carne y leche. Además, sobresalen los servicios como transporte terrestre y marítimo, bancos, hospitales y demás, así como el comercio de tiendas, 
panaderías, supermercados, etc.; y el turismo, tanto de nacionales como extranjeros. Es importante indicar que el muelle de Puntarenas recibe cruceros por temporadas, facilitando la visita de extranjeros a la ciudad (Municipalidad de Puntarenas).

De acuerdo con la información suministrada por Instituto Costarricense de Puertos del Pacífico (INCOP), el puerto de Puntarenas llego a ser uno de los más importantes entre los años 1814 a 1982, a partir del año 1996, por medio de una donación del gobierno de la República de China, se desarrolló una mejor capacidad para la atención de los turistas provenientes de cruceros y de los visitantes en general, así como también, este aporte permitió la construcción la Plaza del Pacífico (Instituto Costarricense de Puertos del Pacífico, INCOP, 2010).

Según lo reportado por el INCOP, la temporada de cruceros en Puntarenas se da por primera vez en el año 2010. Para esta fecha se esperaba la llegada de por lo menos 130 cruceros, con la expectativa de que esto aumentaría la visita, tanto de extranjeros como nacionales, y se planearon una serie de eventos con grupos artísticos y actividades culturales (Instituto Costarricense de Puertos del Pacífico, INCOP, 2010). Hasta la fecha se reciben alrededor de 145 cruceros por año.

\section{ANTECEDENTES}

Es importante destacar el estudio de elementos de política para un turismo de cruceros sostenible en Centroamérica, elaborado por el Centro para Ecoturismo y Desarrollo Sostenible, del Instituto Centroamericano de Administración de Empresas (INCAE) (Centro para Ecoturismo y Desarrollo Sostenible, INCAE, 2007), en el que se investigaron los impactos económicos, socioculturales y ambientales del turismo de cruceros. Esta investigación se llevó a cabo en Costa Rica y Honduras y sirvió para establecer estrategias de desarrollo del turismo de cruceros en Centroamérica. En relación con el caso de Costa Rica, menciona que los visitantes provenientes de los cruceros son atraídos por la naturaleza y la cultura; muchos extranjeros que llegan al país tiene como objetivo primordial conocer sus bosques, playas y volcanes (Centro para Ecoturismo y Desarrollo Sostenible, INCAE, 2007).

Este estudio establece algunas sugerencias importantes que tienen como fin maximizar las divisas obtenidas a través de este tipo de actividad turística, entre las cuales se puntualizan las siguientes: aumentar la tasa de desembarque, ya que en Costa Rica es del $60 \%$,mientras que en otros países es del 85\%, como en Belice; además, aumentar la tasa del impuesto por pasajero: en Costa Rica es $\$ 2,50$, en comparación con la de otros países, como algunos del Caribe, que en promedio tienen una tasa de $\$ 8,66$. Otra propuesta apunta a la ampliación de los contratos de los cruceros, en lo que se refiere a los operadores terrestres, ya que actualmente se 
encuentran en manos de unos pocos empresarios que se benefician de la actividad. Y finalmente, se sugiere el cobro de una contribución a cada turista para proteger los recursos naturales y culturales.

Por otra parte, esta investigación menciona que en Honduras el turismo de los cruceros, si bien es cierto, ha producido empleo para la población, los habitantes más pobres no se han visto tan favorecidos, ya que las mayores ganancias se concentran en pocas empresas, por consiguiente en pocas manos. En Costa Rica, al igual que en Honduras, los mayores beneficios se concentran en pocos operadores de paquetes turísticos, por lo que se recomienda capacitar a la población local para que se involucren en este tipo de turismo y también tenga la posibilidad de ofrecer a los visitantes paquetes turísticos que cumplan con requisitos mínimos de calidad y seguridad (Centro para Ecoturismo y Desarrollo Sostenible, INCAE, 2007).

Al tomar en cuenta este estudio, es importante destacar que en el análisis FODA (Fortalezas, Oportunidades, Debilidades y Amenazas), llevado a cabo en Costa Rica en relación con el turismo de cruceros, se encontraron como fortalezas: la cercanía de los bosques y demás atractivos naturales, la infraestructura portuaria, la poca probabilidad de que el país se vea afectado por huracanes, etc. Entre las oportunidades se mencionan: aumentar la tasa de los pasajeros que desembarcan y mejorar la condición económica de la población, siempre y cuando hayan reformas políticas e institucionales. Se señalan como debilidades la lejanía respecto a Estados Unidos, la calidad de las playas y que no existan muchas atracciones en los puertos (Centro para Ecoturismo y Desarrollo Sostenible, INCAE, 2007).

Como se menciona en este estudio, las líneas de cruceros deberían distribuir en forma equitativa sus ganancias y los gobiernos también deben procurar una mejor negociación con las navieras con el fin de obtener mayores beneficios; además se debe contar con políticas oficiales que garanticen el intercambio comercial entre los pasajeros y los vendedores locales. De esta manera se verá favorecida la población local (Centro para Ecoturismo y Desarrollo Sostenible, INCAE, 2007).

Otro estudio relacionado con el turismo en Puntarenas es Puntarenas y el turismo ¿Que ha pasado con la "perla del Pacífico"?, el cual tiene que ver con el desarrollo de la zona de Puntarenas. Considera distintos elementos como la historia, la economía, las actividades económicas, los lugares a visitar y cómo ha sido el desarrollo del turismo en la zona. Según Chen y García (Chen Mok \& García Cousin, 2007, págs. 127-128) debe existir una mejor organización por parte de la comunidad, las empresas y la municipalidad con el fin de tener un plan para que Puntarenas sea y se mantenga como una atracción para todos los turistas, además, el turista extranjero a transformado a Puntarenas como un lugar donde solo está de paso, ya que no permanece en el sitio, sino que trasborda hacia otros lugares. 


\section{FUNDAMENTOS TEÓRICOS}

El turismo constituye una fuente de ingresos económicos para las regiones dedicadas a esta actividad, siempre y cuando se encuentre bien organizada, de forma que los beneficios que se obtengan favorezcan a distintos sectores. De acuerdo con la Enciclopedia Práctica Profesional de Turismo, Hoteles y Restaurantes, la industria turística es la que se encarga de la atención de los turistas y debe considerar aspectos como transporte, alimentación y distracción (Gispert, 2000).

El turismo contribuye positivamente con el desarrollo de diferentes sectores sociales, de forma directa e indirectamente, por ejemplo, en el caso de la actividad relacionada con el arribo de cruceros a la provincia de Puntarenas, es posible que esté beneficiando directamente a agencias turísticas de nuestro país e indirectamente o en forma secundaría, a otras empresas o comercios afines. Para que el turismo de una región sea exitoso, debe ser promovido por varias instancias, en este sentido los gobiernos locales juegan un papel muy importante en la gestión de esta actividad, como se menciona, las oficinas gubernamentales deben tomarse en cuenta como integrantes importantes en este particular (Gispert, 2000).

Así, el turismo es muy importante para la sociedad, ya que representa el empleo del tiempo libre en diferentes actividades, que sugieren bienestar y disfrute para las personas. El turismo en el nivel social se entiende como una dinámica relacionada con placer, descanso, desarrollo cultural y recreación (Acerenza, 1991).

La práctica del turismo trae bienestar a las personas, ya que experimentan diversos sentimientos y emociones mientras disfrutan periodos de vacaciones, y además genera ventajas para el país que lo promueve, en diferentes lugares y por medio de actividades variadas, las cuales se organizan como parte de la industria turística, según Acerenza. Los beneficios para los países promotores se manifiestan de formas muy diversas, ya que esta actividad ayuda a aumentar el producto interno; así por ejemplo, se pueden obtener ingresos que contribuyan al pago de salarios de las personas involucradas (Acerenza, 1991).

Por otra parte, también se generan otras formas de producción, como la compra de un producto interno, o sea la adquisición de productos para comerciar con los turistas. Un ejemplo claro de esto es el comercio que se da entre los restaurantes y los intermediarios, éstos últimos negocian directamente con agricultores de la zona; lo que se traduce en una colaboración específica el sector agrícola; y lo mismo sucede con otros sectores. Otro punto importante es la contribución del turismo a la redistribución del ingreso, ya que las ganancias producidas en esta industria se redistribuyen posteriormente entre la población, así que, tomando en cuenta las zonas que se destinan para el turismo en las diferentes poblaciones y los ingresos que se generan de la actividad turística, se perciben beneficios económicos para las regiones donde están ubicadas (Acerenza, 1991). 
El turismo produce empleos, lo cual va a depender de la importancia que cada país le de a esta actividad. Lo anterior va relacionado con el producto interno de las regiones, ya que a menor producto, menor empleo. Por otra parte, en los países donde el turismo toma relevancia como fuente significativa de ingresos, consecuentemente va a generar más ingresos (Acerenza, 1991).

Cabe destacar que el turismo se ve afectado por las condiciones favorables o desfavorables de una región, así por ejemplo, existen desigualdades en ciertas regiones, que en lugar de incrementar la actividad turística, más bien marcan claras diferencias en comparación con otras zonas, lo que afecta su desarrollo económico.

De acuerdo con Acerenza (1991), existen diferentes teorías que ayudan a explicar el porqué se dan las desigualdades regionales. La primera es la teoría de las aglomeraciones, la cual consiste en una región que concentra empresas o plantas industriales en una misma ubicación, lo que implica requerimientos de infraestructura elemental, servicios básicos de energía y agua, existencia de un sistema vial y de las comunicaciones. Lo anterior proporciona ventajas, pues ayuda a desarrollar un mercado, produce más trabajo y demás; sin embargo afecta una región en el sentido de que se continuará con la aglomeración aun cuando los espacios físicos ya estén muy reducidos. Una situación de este tipo causa, por ejemplo, que haya menos terrenos disponibles, incremento en el precio de las propiedades y aumento en los precios de las rentas de las casas; así como en los servicios públicos, entre otras consecuencias. Dado lo anterior, el turismo de una región se ve afectado, ya que los problemas sociales y políticos se intensifican, y se produce una marcada desigualdad respecto a otras regiones.

Otra teoría de la desigualdad de las regiones, planteada por el mismo autor, se refiere a la teoría de los costos sociales, entendidos estos y derivados de las aglomeraciones de empresas y de población, en puntos específicos en las regiones. Esto trae como consecuencia lo que se denomina como costos sociales, tales como la contaminación, los malos olores, la basura en los espacios públicos, etc., lo que repercute directamente en el desarrollo del turismo en las regiones.

También, se propone la teoría de la dominación, que tiene relación con las diferencias que existen entre las partes del mercado y el dominio que ejercen unas sobre otras, ya que el sector dominante va a beneficiarse más que otros. Lo anterior se vincula con el poder que tienen cada una de las partes, pues algunas tienen mucha más participación en los mercados y es así como las llamadas "dominantes" se consideran privilegiadas y son las activas del medio, mientras que las otras adoptan el papel de pasivas. En consecuencia, mientras las dominantes son sinónimo de progreso, las pasivas representan un estancamiento.

Finalmente se agrega la teoría de las instituciones sociales, la cual se relaciona con los grupos sociales que habitan en regiones donde el estancamiento 
económico y la aglomeración son el común denominador. A partir de, esta propuesta se establecen dos grandes sociedades: la progresista y la tradicional. En esta última ocurre un estancamiento y prevalecen las costumbres tradicionales, Estos pequeños grupos mantienen su autoridad y así sostienen el predominio de sus intereses, lo que trae como consecuencia las desigualdades de las regiones (Acerenza, 1991).

Estas teorías tienen relación con la investigación propuesta del turismo de cruceros en la ciudad de Puntarenas y precisamente en este estudio se logra determinar si existen o no desigualdades en esta región, tomando en cuenta las limitaciones y los problemas detectados. La solución a estas situaciones podría beneficiar mayormente este tipo de turismo en la provincia. Además, los resultados muestran como la teoría de la dominación puede explicar el hecho de que unos grupos se benefician más que otros, pues algunos tienen más posibilidades de vender sus productos en los cruceros, ya que están más organizados con las compañías navieras que los traen al país.

\section{METODOLOGÍA}

La presente investigación toma en cuenta el enfoque cualitativo, pues se plantea un problema al investigar el mundo social (Hernández, Fernández y Baptista, 2006), se recolectan una serie de datos y las opiniones de los participantes son muy importantes para el análisis de los resultados; también se toma en cuenta el enfoque cuantitativo, pues los datos se deben agrupar utilizando estadísticas.

Las características de orden cualitativo se evidencian en el uso de la recolección de datos sin medición numérica, sin datos estadísticos. Por otra parte, las de tipo cuantitativo, se cumplen cuando se analizan datos contables con medición numérica, en este caso las variables planteadas en la investigación, como se afirma en Hernández et. al (2006) : "el investigador recolecta datos numéricos de los objetos, fenómenos o participantes, que estudia y analiza mediante procedimientos estadísticos".

Cabe destacar que en este estudio se utilizaron herramientas como la entrevista, el cuestionario y la revisión de documentos, por lo tanto, los dos enfoques, tanto el cualitativo como el cuantitativo, están presentes en la investigación.

La entrevista cualitativa fue uno de los métodos más efectivos para este trabajo, pues permitió tener una visión más general de las diferentes variables planteadas, así como recolectar información muy necesaria para la interpretación de los resultados. 


\section{MUESTRA}

Primero se indagó acerca de las instituciones que tienen que ver con el turismo de cruceros en la provincia de Puntarenas, entre las que destacan: el Instituto Costarricense de Turismo, la Cámara de Turismo de Puntarenas, la Junta Promotora de Turismo del Instituto Costarricense de Puertos del Pacífico (INCOP), la Municipalidad de Puntarenas, etc.; y se logró concretar entrevistas con las autoridades pertinentes.

También se identificaron diferentes negocios y personas que se veían afectadas directa o indirectamente con el turismo de cruceros en la provincia de Puntarenas. La muestra se conformó con la participación de 55 personas, entre ellos, dueños de restaurantes, tiendas, puestos de "souvenirs" (artículos de recuerdo de la visita al país), licoreras, vendedores ambulantes de comidas preparadas. Básicamente se entrevistó a todo el sector comercial ubicado a lo largo del Paseo de los Turistas.

\section{RESULTADOS}

Para valorar los resultados se consideraron los mecanismos de la entrevista y el cuestionario.

Se entrevistó a la directora de la Junta Promotora de Turismo del INCOP, señora Ileanette Canesa Alfaro, acerca de cuál es el impacto social y económico generado por el turismo de los cruceros. La funcionaria opina que sí ha habido un impacto positivo. Agrega que la organización que dirige se encarga de los planes de mercadeo, y que se han mejorado los servicios, aunque todavía falta más apoyo por parte de la ciudadanía y otras instituciones. Considera que las personas se deben involucrar más y también, que se deben crear más fuentes de empleo.

Uno de los aspectos que puntualiza es la necesidad de captar más turistas y de que éstos permanezcan más tiempo en la ciudad, ya que menciona que solo un $40 \%$ del turista que viene en los cruceros se queda en la provincia, los demás se van en los buses en tours o se quedan dentro del barco.

En cuanto a la interrogante sobre las actividades que se le ofrecen al turista, menciona que se hacen actividades con música y bailes, además, el muelle se decora. También se les envía información a los cruceros sobre las actividades y lugares turísticos que tiene la zona, para promover las visitas de los turistas a la provincia.

Al consultarle sobre las dificultades que se les presentan, la directora menciona que uno de los impedimentos es que muchas de las personas que quieren vender productos no cuentan con permisos, lo que se convierte en un problema. Agrega que es necesario involucrar a diferentes instituciones y a la comunidad, así 
como capacitar a las personas que se relacionan directa o indirectamente con este tipo de turismo.

Respecto a las necesidades que ella considera es importante solventar, menciona que todavía falta mucha infraestructura turística y promocionar la inversión extranjera. Comentó que se tienen proyectos por desarrollar, como mejorar el muelle para traer más cruceros.

En cuanto a la gestión de la Junta Promotora explica que se trabaja en campañas promocionales de mercadeo y en estrategias para negociar con agencias navieras para establecer nuevas rutas que tomen en cuenta a Puntarenas como destino. Destaca que en la actualidad se reciben más o menos 145 cruceros por año y que se busca implementar nuevas tácticas y mejorar las condiciones, para duplicar la cantidad de cruceros.

Explica que una parte de los impuestos que se cobran por la entrada de los cruceros a los puertos, es administrada por el INCOP y la Municipalidad. Aclara que el INCOP tiene un superávit que se destina a los proyectos en la comunidad, como por ejemplo el balneario nuevo ubicado en la punta del puerto, que, como ella menciona, es un proyecto de $\$ 3$ millones; además, la pintura de murales en la Clínica San Rafael, ubicada en el Paseo de los Turistas y en otros lugares del cantón central; arreglos a los parques: Lobo, Chacharita y Esparza, malecones, canchas, parques infantiles; entre otros. Agrega que en una segunda etapa se pretende construir más malecones, un pequeño muelle y un faro ${ }^{1}$.

También se entrevistó a la señora Marny Chan, Directora de Hacienda de la Municipalidad de Puntarenas, quien explica que la municipalidad recibe $\$ 1,50$ por cada pasajero que tenga movimiento en el muelle de Puntarenas. Afirma que el dinero recaudado en los cruceros se destina a la limpieza y ornato, seguridad, maquinaría para limpieza de playa, pago de cuadrillas de limpieza de playas, combustible, compra de bolsas de basura, entre otros. Menciona que el monto que recibe la Municipalidad se destina solamente para estos rubros, ya que no alcanza para más y los ingresos que administra el INCOP se utilizan para llevar a cabo otros proyectos ${ }^{2}$.

De acuerdo con la información que suministra el Instituto Costarricense de Puertos del Pacífico (INCOP), los atractivos turísticos de la zona son los siguientes: Muelle Grande, Mercado De Puntarenas, Parque Mora y Cañas, La Catedral, Casa de la Cultura, Parque Marino, Reserva Cabo Blanco, Estero Mata Limón, entre otros; también promociona las comidas propias de la zona como ceviches, arroz con calamares, mieles, etc. (2010)

Según el estudio realizado en los diferentes puestos de venta de comida como restaurantes, heladerías, quioscos y en puestos de artesanías; cuando llegan cruceros a la ciudad de Puntarenas, la cantidad de sus clientes aumenta moderadamente, por 
lo tanto esta fue la opción elegida por la mayoría de las personas, sin embargo, aclararon que el incremento en las ventas dependía de las personas de los cruceros, ya que algunos turistas compraban más que otros, por lo que califican el aumento de sus ingresos como moderado.

Porcentualmente, al aumento de los ingresos económicos por la visita de cruceros a la ciudad de Puntarenas, se determino de acuerdo a la información suministrada por la mayoría de las personas entrevistadas, quienes opinan que hay un incremento moderado de sus clientes y por consiguiente de sus ingresos económicos. Esto está representado con un $42 \%$ de la muestra. Un 35\% opina que la visita de los cruceros y los turistas aumenta muy poco la cantidad de clientes y sus ingresos económicos, un $18 \%$ dice que no aumenta en lo más mínimo la cantidad de clientes e ingresos económicos y finalmente un $5 \%$ opina que aumenta considerablemente la cantidad de clientes y de ingresos económicos. Es importante destacar que muchas personas que cooperaron con este estudio coincidieron en sus apreciaciones, ya que opinaron que, de acuerdo al producto que se ofrece, la venta se incrementa y por consiguiente los ingresos económicos, otro factor que se mencionó fue la coyuntura de cada temporada y afirmaron que la vigente en el momento del estudio estaba muy baja. Entre los productos que más se venden, según los entrevistados, destacan: recuerdos, licor y tabaco; mientras que los alimentos no se venden mucho, ya que el crucero incluye la alimentación.

Cabe destacar que muchas de las personas entrevistadas opinan que el turismo nacional es el que consume en mayor cantidad los diferentes productos que ellos ofrecen, sin embargo, no es todo el tiempo, pues mencionan que su clientela aumenta un poco más los fines de semana.

A continuación se muestra una figura donde se representa el porcentaje, de acuerdo al aumento de la cantidad de clientes e ingresos económicos.

En cuanto a la pregunta relacionada con el mejoramiento de la actividad económica de los porteños en general, por la visita de los cruceros a la zona, un $42 \%$ determinó que ha mejorado muy poco la actividad y condición económica de los pobladores de la zona. Entre las razones mencionadas destacan que la mayoría de las personas que vienen en los cruceros no se quedan en la zona, más bien ya tienen programados tours a otros lugares más alejados de Puntarenas, que ya están incluidos en los paquetes de viaje, por lo cual les gustaría que más visitantes permanecieran en el puerto. Otra causa es que la mayoría de los turistas no consumen los productos que ofrecen, no compran artesanías u otras mercancías, según los entrevistados, hoy día es más difícil vender, ya que el turista muchas veces solicita rebajas en los precios, lo que es muy difícil para los vendedores, pues las materias primas con las que trabajan son muy caras en Costa Rica, por lo tanto, vender muy barato no les permite obtener ganancias. Los vendedores que tienen puestos de 
recuerdos explican que en otros países si les hacen rebajas de precios a los turistas y ellos creen que esa es la razón para que les soliciten lo mismo, pero como se mencionó anteriormente, no es posible bajar los costos dados los precios de las materias primas en el país.

Tabla 1

C ONSIDERACIONES ACERCA DE LOS MOVIMIENTOS DE LA CANTIDAD DE CLIENTES Y DE LOS INGRESOS ECONÓMICOS DURANTE LA VISITA DE LOS CRUCEROS;

EN VALORES ABSOLUTOS Y RELATIVOS

\begin{tabular}{lcc}
\hline \multicolumn{1}{c}{ Consideración } & Absolutos & Relativos (\%) \\
\hline $\begin{array}{l}\text { Aumenta moderadamente la cantidad de sus clientes y } \\
\text { por consiguiente sus ingresos económicos }\end{array}$ & 23 & $42 \%$ \\
$\begin{array}{l}\text { Aumenta muy poco la cantidad de sus clientes y por } \\
\text { consiguiente sus ingresos económicos }\end{array}$ & 19 & $\mathbf{3 5 \%}$ \\
$\begin{array}{l}\text { No aumenta la cantidad de sus clientes ni de sus } \\
\text { ingresos económicos }\end{array}$ & 10 & $\mathbf{1 8 \%}$ \\
\hline $\begin{array}{l}\text { Aumenta considerablemente la cantidad de sus } \\
\text { clientes y por consiguiente sus ingresos económicos }\end{array}$ & 3 & $\mathbf{5 \%}$ \\
\hline \begin{tabular}{l} 
Total \\
\hline
\end{tabular} & $\mathbf{5 5}$ & $\mathbf{1 0 0 \%}$ \\
\hline
\end{tabular}

Otra causa que se señaló fue el hecho de que las personas consideran que ha disminuido la visita de embarcaciones, ya que ellos dicen que en el pasado más cruceros visitaban la zona. Lo anterior motivado por problemas como la infraestructura portuaria deficiente, la inseguridad, la falta de un lugar más atractivo para ofrecer los productos, la falta de actividades y sitios cercanos que atraigan más al turista y lo motiven para quedarse en la zona. 

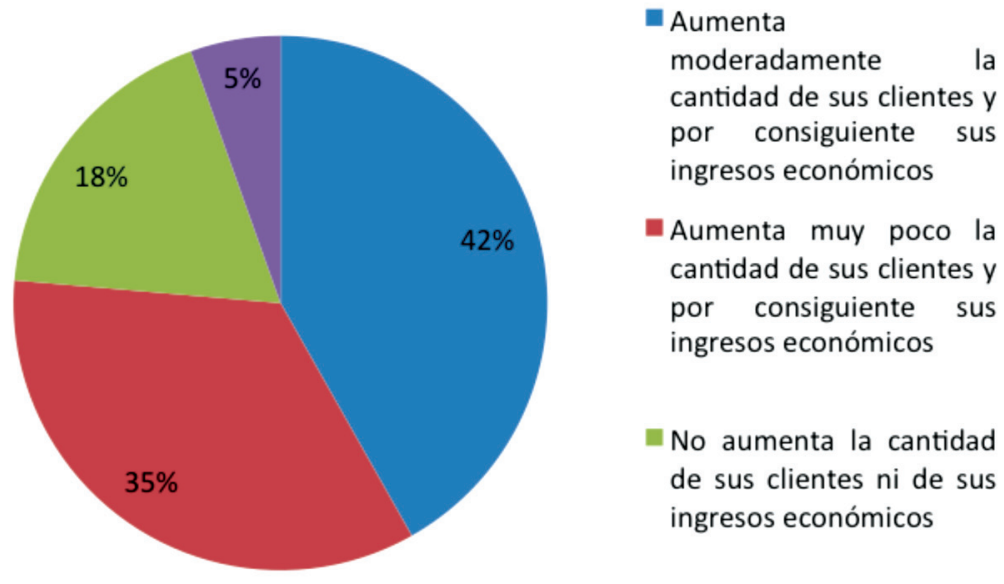

Figura 2. Consideraciones acerca de los movimientos de la cantidad de clientes y de los ingresos económicos durante la visita de los cruceros; en valores relativos.

Un $40 \%$ opinó que la visita de los cruceros ha mejorado moderadamente la actividad económica de los porteños, pues proporciona empleo a los pobladores de la zona, un $15 \%$ dice que no ha mejorado la actividad económica de los porteños y ninguno opinó que la ha mejorado considerablemente al ofrecer mucho empleo. A continuación se representan los resultados gráficamente:

Tabla 2

OPINIÓN DE LOS ENTREVISTADOS RESPECTO DE CÓMO EL TURISMO DE LOS CRUCEROS HA AFECTADO LA ACTIVIDAD ECONÓMICA DE LOS PORTEÑOS OFRECIENDO O NO EMPLEO A LOS LUGAREÑOS; EN VALORES ABSOLUTOS Y RELATIVOS

\begin{tabular}{lcc}
\hline \multicolumn{1}{c}{ Variables } & Absolutos & Relativos (\%) \\
\hline $\begin{array}{l}\text { Ha mejorado moderadamente la actividad económica de } \\
\text { los porteños, ofrece empleo moderado a los lugareños }\end{array}$ & 22 & 40 \\
$\begin{array}{l}\text { Ha mejorado muy poca la actividad económica de los } \\
\text { porteños, ofrece muy poco empleo a los lugareños }\end{array}$ & 25 & 45 \\
$\begin{array}{l}\text { No ha mejorado la actividad económica de los porteños; } \\
\text { no ofrece empleo }\end{array}$ & 8 & 15 \\
$\begin{array}{l}\text { Ha mejorado considerablemente la actividad económica } \\
\text { de los porteños ofreciendo empleo a los lugareños }\end{array}$ & 0 & 0 \\
\hline Total & $\mathbf{5 5}$ & $\mathbf{1 0 0}$ \\
\hline
\end{tabular}




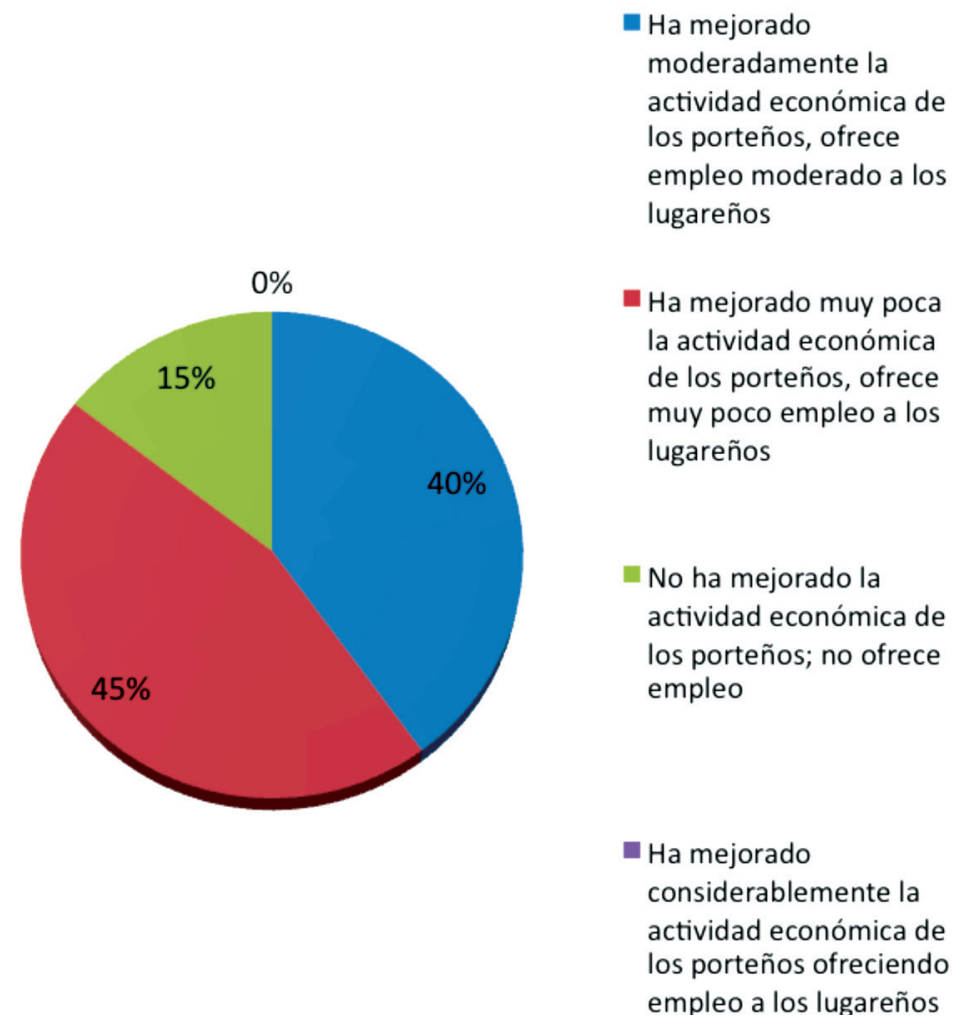

Figura 3. Opinión de los entrevistados respecto de cómo el turismo de los cruceros ha afectado la actividad económica de los porteños ofreciendo o no empleo a los lugareños; en valores relativos.

En cuanto a las acciones que se realizan para atraer al turista, tanto nacional como internacional, un $45 \%$ de los colaboradores opinó que existe muy poca promoción para atraerlos, un $42 \%$ dice que no existe ninguna promoción para atraer al turista, un $13 \%$ opina que se da una moderada promoción para atraer a los visitantes y ningún entrevistado opinó que se da mucha promoción.

A continuación se representa gráficamente el porcentaje de personas que opinaron en cuanto a las actividades que se realizan en temporada de cruceros.

Tomando en cuenta las posibles limitaciones encontradas, los colabores de la investigación opinaron, en un $34 \%$, que uno de los mayores problemas es la falta de interés para mejorar aspectos como la infraestructura en la zona , un $33 \%$ opinó que uno de los principales problemas es la necesidad de promover más proyectos y actividades; y la creación de lugares atractivos cerca de la ciudad de Puntarenas con el fin de que el turista, tanto nacional como internacional, se sientan más atraído a visitarla. Un 33\% dice que una de las limitaciones más importantes de la región es la falta de empleo, pese a la visita de los cruceros. 


\title{
Tabla 3
}

CRITERIO DE LOS ENTREVISTADOS RESPECTO DE SI SE HAN REALIZADO O NO ACCIONES CON

MUCHA, MODERADA, MUY POCA O NINGUNA PROMOCIÓN PARA ATRAER

AL TURISTA NACIONAL Y EXTRANJERO A LA ZONA DE PUNTARENAS;

EN VALORES ABSOLUTOS Y RELATIVOS

\begin{tabular}{lcc}
\hline \multicolumn{1}{c}{ Alternativa } & Absolutos & Relativos (\%) \\
\hline $\begin{array}{l}\text { Muy poca promoción para atraer al turista nacional } \\
\text { e internacional }\end{array}$ & 25 & 45 \\
$\begin{array}{l}\text { Ninguna promoción para atraer al turista nacional } \\
\text { e internacional }\end{array}$ & 23 & 42 \\
$\begin{array}{l}\text { Moderada promoción para atraer al turista nacional } \\
\text { e internacional }\end{array}$ & 7 & 13 \\
$\begin{array}{l}\text { Mucha promoción para atraer al turista nacional } \\
\text { e internacional }\end{array}$ & 0 & 0 \\
\hline Total & $\mathbf{5 5}$ & $\mathbf{1 0 0}$ \\
\hline
\end{tabular}

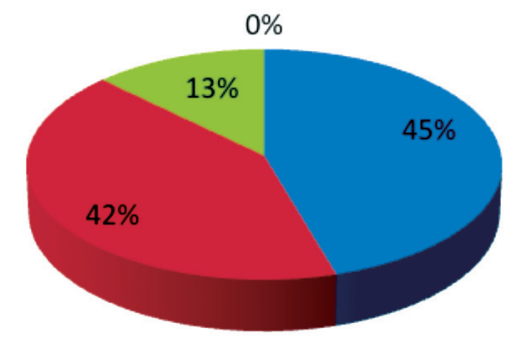

\author{
Muy poca promoción \\ para atraer al turista \\ nacional e \\ internacional \\ - Ninguna promoción \\ para atraer al turista \\ nacional e \\ internacional \\ Moderada promoción \\ para atraer al turista \\ nacional e \\ internacional \\ - Mucha promoción para \\ atraer al turista \\ nacional e \\ internacional
}

Figura 4. Criterio de los entrevistados respecto de si se han realizado o no acciones con mucha, moderada, muy poca o ninguna promoción para atraer al turista nacional y extranjero a la zona de Puntarenas; en valores relativos.

Es importante destacar que en esta pregunta los colaboradores podían elegir las tres opciones, si ellos consideraban que las tres eran limitantes o problemas de la zona. A continuación la representación gráfica: 


\section{Tabla 4}

POSIBLES LIMITACIONES ENCONTRADAS ACERCA DE LOS PROBLEMAS ECONÓMICOS Y SOCIALES QUE ENFRENTA LA PROVINCIA PUNTARENENSE; EN VALORES

ABSOLUTOS Y RELATIVOS

\section{Consideración}

Absolutos

Relativos (\%)
Falta de apoyo e interés por parte de autoridades para mejorar más infraestructura en esta zona

Falta por parte de las autoridades para promover más proyectos, actividades y lugares más atractivos de la zona para el turista nacional e internacional

Falta de empleo en la zona pese a la visita de los cruceros

Otra
44

0

128
34

33

33

0

Total 100

- Falta de apoyo e interés del gobierno para mejorar más infraestructura en esta zona

- Falta por parte de las autoridades pertinentes para promover más actividades y lugares más atractivos de la zona para el turista nacional e internacional

Figura 5. Posibles limitaciones encontradas acerca de los problemas económicos y sociales que enfrenta la provincia puntarenense; en valores relativos.

Entre las justificaciones que se dieron para la respuesta de que existe muy poca promoción para atraer al turista, o no existe del todo, se destacan: La falta de organización de más actividades para que los turistas, y crear más atractivos en la zona para que permanezcan más tiempo en el lugar. Además, mencionan que no se hace mucha promoción de los sitios que podrían ser del agrado de los turistas y que se encuentran muy cerca o en la propia ciudad de Puntarenas. Agregan que es 
necesario ofrecer capacitación a los lugareños para que se encarguen de hacer tours u otras actividades que les generen más empleo.

También se investigó por medio de preguntas abiertas cuáles son las actividades que se realizan en Puntarenas cuando hay visitas de cruceros, entre las respuestas tenemos que 35 personas opinaron que no se hace ninguna actividad y 20 personas manifestaron que se realizan ciertas actividades, aunque no todo el tiempo, como las siguientes:

- Un bus que recorre la ciudad de Puntarenas con los turistas.

- Bailes típicos, mascaradas y degustación de granizados.

- Desfile de Bandas de escuelas en la ciudad de Esparza.

Entre las actividades que se organizan regularmente en la temporada de cruceros destacan:

- Puestos con ventas de artesanías.

- Se dispone de un tren en el muelle para trasladar a los turistas hacia la ciudad.

- Tours a diferentes sitios turísticos del país como a las playas Jacó y Tárcoles,

Parques Nacionales, el cantón de Sarchí, etc., los cuales forman parte del paquete del crucero que venden con anterioridad las agencias de viajes.

A continuación se representa gráficamente el porcentaje de personas que opinaron en cuanto a las actividades que se realizan durante la temporada de cruceros.

\section{Tabla 5}

CONSIDERACIÓN DE LOS ENTREVISTADOS ACERCA DE SI SE REALIZAN ACTIVIDADES DURANTE LA TEMPORADA DE CRUCEROS EN PUNTARENAS;

EN VALORES ABSOLUTOS Y RELATIVOS

\begin{tabular}{ccc}
\hline Opinión & Absoluto & Relativo \\
\hline Sí & 35 & $64 \%$ \\
No & 20 & $36 \%$ \\
\hline Total: & $\mathbf{5 5}$ & $\mathbf{1 0 0 \%}$ \\
\hline
\end{tabular}




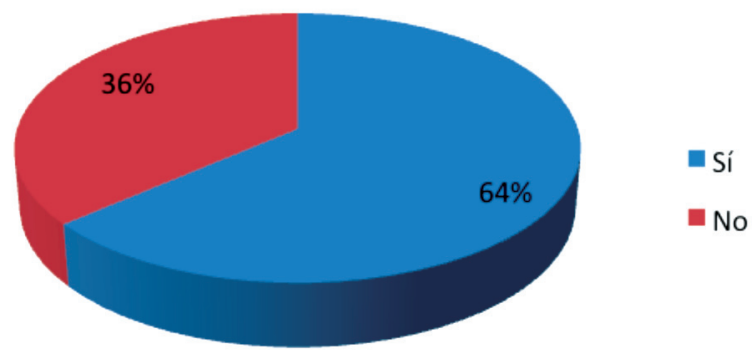

Figura 6. Consideración de los entrevistados acerca de si se realizan actividades durante la temporada de cruceros en Puntarenas; en valores relativos.

Otra interrogante se relacionó con las posibles soluciones a los problemas planteados. Entre las propuestas planteadas por los entrevistados se mencionaron:

Tabla 6.

POSIBLES SOLUCIONES A LOS PROBLEMAS PLANTEADOS.

Que desarrollen más proyectos turísticos en la zona

Mejorar más en infraestructura

Más interés y compromiso por parte del autoridades y empresas.

Que se desarrollen más actividades para atraer tanto al turismo nacional como el extranjero

Mayor organización entre las autoridades

Motivar a los turistas a comprar más productos de la zona

Que el costo de los productos y entradas a lugares turísticos no sea tan elevado.

Reactivar el programa de bienvenida de los cruceros, que sea permanente.
Más información y promoción a los atractivos de Puntarenas

Que no se lleven a los turistas de cruceros en tours fuera de la ciudad

Más participación de la comunidad en diversas actividades

Más seguridad y limpieza y más zonas de parqueo.

Embellecer el paseo con ranchos, más pollitos, puestos para venta de artesanías

Que se beneficie más la población local

Más fuentes de trabajo

Más capacitaciones

Que se promocionen más los productos que se pueden adquirir en la zona. 


\section{CONCLUSIONES}

- La mayoría de los colaboradores de la investigación determinaron que sus ingresos económicos aumentan moderadamente cada vez que hay visita de cruceros, muchos coincidieron que este incremento depende de la temporada y del producto que comercian, ya que hay épocas en que llegan muchos barcos de este tipo y además no siempre los visitantes se quedan en la zona. Por otra parte, no consumen muchos de los alimentos que se venden en el Puerto.

- $\quad$ Entre los productos que más se venden se mencionaron: recuerdos, licor y tabaco.

- Los turistas nacionales que visitan la zona son los que más consumen los productos ofrecidos, especialmente los fines de semana.

- En cuanto al aumento de la actividad y condición económica de los porteños, en el sentido de que el turismo de los cruceros genera más empleo, la mayoría de los colaboradores opinó que había aumentado muy poco. Entre las razones que se manifestaron, destacan: que las agencias grandes, que tienen contactos con las compañías de cruceros, ya tienen los paquetes turísticos vendidos, por lo que el visitante no se queda en la zona, sino que realiza "tours" en regiones alejadas; además también se menciona que este tipo de turistas no consumen mucho los productos que los lugareños venden; otra causa es que, según los entrevistados, han disminuido las embarcaciones que llegan al Puerto, pues antes había una mayor afluencia, y finalmente señalan problemas como inseguridad, poca limpieza, infraestructura deficiente, etc.

- La mayoría de los entrevistados consideró que existen muy pocas actividades de promoción para atraer al turista, tanto nacional como extranjero, por lo que, en general se considera que se debe promocionar mucho más la región, tanto fuera como dentro del país.

- Para la mayoría de los participantes en la muestra, la limitación o problema que más sobresale es la falta de apoyo e interés por parte de las autoridades para mejorar la infraestructura, en segundo lugar está la necesidad de promover más actividades y acondicionar lugares que atraigan al turista; y finalmente, la falta de empleo.

- $\quad$ En cuanto a las actividades que se realizan, la mayoría opina que sí se realizan actividades, pero que estas son esporádicas, por lo que se deben efectuar de forma regular y en mayor cantidad, según su criterio.

- $\quad$ Entre las soluciones más relevantes a los problemas planteados sobresalen: mayor desarrollo de proyectos turísticos en la zona, mejorar la infraestructura, mayor interés y compromiso por parte de las autoridades y empresas, 
para que no se lleven a los turistas de cruceros a "tours" fuera de la ciudad, con el fin de que se beneficie la población local; embellecer el paseo con ranchos, más asientos puestos para venta de artesanías, etc.

- De acuerdo con la entrevista realizada a la directora de la Junta Promotora de Turismo del INCOP se han desarrollado muchos proyectos como el balneario, ubicado en la punta, los murales pintados en la Clínica San Rafael, ubicada en el Paseo de los Turistas y otros lugares de la ciudad, arreglos en los parques: Lobo, Chacharita y Esparza, malecones, canchas de baloncesto y parques infantiles, entre otros; además se planea continuar con una segunda etapa para construir más malecones y hacer otras mejoras.

\section{CITAS Y NOTAS}

1 Entrevista realizada a Directora de Junta Promotora de Turismo: Ileanette Canesa Alfaro.

2 Entrevista realizada a Marny Chan, Directora de Hacienda de la Municipalidad de Puntarenas.

\section{BIBLIOGRAFÍA}

Acerenza, M. Á. (1991). Administración del Turismo: Conceptualización y Organización. México, México: Trillas.

Centro para Ecoturismo y Desarrollo Sostenible, INCAE. (2007). Elementos de política para un turismo de Cruceros sostenible en Centroamérica. Resumen para quienes toman decisiones.

Chen Mok, S., \& García Cousin, K. (2007). Puntarenas y el turismo: ¿Qué ha pasado con la «Perla del Pacífico»? Inter Sedes, VIII(15).

Gispert, C. (2000). Enciclopedia Práctica Profesional de Turismo, Hoteles y Restaurantes. España: Grupo Editorial Oceáno.

Hernández, Fernández y Baptista. (2006). Metodología de la Investigación. McGraw-Hill.

Instituto Costarricense de Puertos del Pacífico, INCOP. (2010). Información Turística. Recuperado el 28 de setiembre de 2012, de http://www.incop.go.cr/la_ciudad_de_puntarenas.php?p=91

Instituto Costarricense de Puertos del Pacífico, INCOP. (2010). Puntarenas y Su Historia. Recuperado el 28 de setiembre de 2012, de http://www.incop.go.cr/la_ciudad_de_puntarenas. $\mathrm{php}$ ? $=91$

Instituto Costarricense de Puertos del Pacífico, INCOP. (2010). Temporada de Cruceros. Recuperado el 30 de setiembre de 2012, de http://www.incop.go.cr/la_ciudad_de_puntarenas. $\mathrm{php}$ ? $=91$ 
Instituto Costarricense de Puertos del Pacífico, INCOP. (2010). Terminal de Cruceros. Recuperado el 28 de setiembre de 2012, de http://www.incop.go.cr/la_ciudad_de_puntarenas.php?p=91

Municipalidad de Puntarenas. (s.f.). Reseña del Cantón de Puntarenas. Recuperado el 1 de octubre de 2012, de http://www.munipuntarenas.go.cr/index.php

\section{ACERCA LA AUTORA}

María Lisbeth Mora Elizondo: La autora es docente en la Sede del Pacífico, Universidad de Costa Rica. Máster en Psicopedagogía, Licenciada en la Enseñanza del inglés, investigadora en temáticas de turismo y educación. 
